Biologia Futura

https://akademiai.com/loi/019

\section{Selection and validation of reference genes for quantitative gene expression analyses in persimmon (Diospyros kaki Thunb.) using real-time quantitative PCR}

Gaigai Du, Liyuan Wang, Huawei Li, Peng Sun, Jianmin Fu, Yujing Suo, Weijuan Han, Songfeng Diao, Yini Mai and

Cite this article: $\mathrm{Du} \mathrm{G}$, Wang L, Li H, Sun P, Fu J, Suo Y, Han W, Diao S, Mai Y, and Li F. 2019. Selection and validation of reference genes for quantitative gene expression analyses in persimmon (Diospyros kaki Thunb.) using real-time quantitative PCR. Biol. Fut. 70, 261-267.

Received: 7 November 2018

Accepted: 20 May 2019

Keywords:

Diospyros kaki Thunb., geNorm, NormFinder, reference genes, RT-qPCR

Key laboratory of Non-timber Forest Germplasm Enhancement \& Utilization of State Forestry Administration, Non-timber Forestry Research and Development Center, Chinese Academy of Forestry, Zhengzhou, China

DOI: $10.1556 / 019.70 .2019 .24$

Background and aims: Persimmon (Diospyros kaki) is an economically important fruit tree species with complex flowering characteristics. To obtain accurate expression pattern analysis results, it is vital to select a reliable gene for the normalization of real-time quantitative polymerase chain reaction data. The aim of this study was to identify the optimal internal control gene among six candidate genes for gene expression analysis in different persimmon organs and developmental stages. Materials and methods: This analysis was conducted using geNorm and NormFinder software to show differences in the stability of the six reference genes among tissues and floral developmental stages of the same plant. Results: Although genes that exhibited moderate expression in NormFinder revealed slightly different expression stabilities than those obtained by geNorm, both sets of results showed that $G A P D H$ was the best reference gene in different organs and floral buds at different developmental stages, whereas $18 \operatorname{SrRNA}$ was the least stable gene. Conclusions: Based on the overall ranking, GAPDH is the most suitable reference gene and is highly recommended for gene expression studies in different organs and different developmental stages of persimmon. This study provides useful reference

data for future gene expression studies and will contribute to improving the accuracy of gene expression results in persimmon.

\title{
INTRODUCTION
}

Persimmon (Diospyros kaki Thunb.) is an economically important fruit tree species in China (Guo \& Luo, 2006). Persimmon is generally classified into four types: pollination constant non-astringent (PCNA), pollination constant astringent (PCA), pollination variant and non-astringent (PVNA), and pollination variant and astringent (PVA; Taira, 1996). PCNA and PVNA fruits are classified as sweet persimmons because they are non-astringent and sweet at harvest maturity, whereas PCA and PVA fruits are considered astringent persimmons based on the presence or absence of astringency in fruit at maturity (Taira, 1996). Most persimmon cultivars are gynoecious; a few are monoecious or androgynomonoecious (such as "Zenjimaru"). Female (pistillate) flowers are usually solitary, and male (staminate) flowers are organized in a three-flower cyme. Thus, research on the regulatory mechanisms of persimmon deastringency and sexuality has generated interest in the scientific community and among economic stakeholders.

Molecular biological techniques are becoming increasingly important in many plant research fields. Understanding gene expression patterns is expected to provide insight into complex regulatory networks and leads to a better understanding of the genes relevant to different biological processes. Among these, real-time quantitative polymerase chain reaction (RT-qPCR) analysis has become a widely used and powerful method for gene expression analysis during plant development due to its accurate quantification, high specificity, and reproducibility. RT-qPCR data interpretation 
largely depends on the stability of internal reference genes applied in data normalization. Reference gene expression stabilities vary among different plant species and tissues, as well as different experimental conditions. For example, actin $(A C T)$ has been ranked as the best internal control for gene expression studies in potato (Nicot et al., 2005) and cineraria (Jin et al., 2013), whereas it is unstable in banana (Chen et al., 2011) and Saccharomyces cerevisiae (Teste et al., 2009). Zhou et al. (2012) found that $A C T$ was the most suitable reference gene for the analysis of different apple tissues, whereas it was unsuitable for comparing samples collected at different developmental stages. Thus, it is vital to assess the expression stability of several candidates before RT-qPCR analysis, to establish a series of reliable reference genes that are expressed constitutively in specific treatments, tissues, and developmental stages.

In recent years, many studies have been conducted on the selection and validation of appropriate genes for data normalization among a wide spectrum of plant species (Chen et al., 2011; Demidenko et al., 2011; Hu et al., 2009; Li et al., 2014; Teste et al., 2009; Tong et al., 2009). Most studies applying RT-qPCR to analyze gene expression in persimmon have used Dkactin as the internal control (Yin et al., 2012; Zhao et al., 2011a, 2011b). However, it remains unclear whether Dkactin is the most suitable gene for research on persimmon because no systematic study has been performed to validate its reference genes.

Therefore, in this study, we selected six candidate reference genes among those used most frequently across a large set of organisms, i.e., $A C T$, glyceraldehyde-3-phosphate dehydrogenase $(G A P D H)$, alpha-tubulin $(T U B), 18 \mathrm{~S}$ ribosomal RNA (18SrRNA), ubiquitin $(U B Q)$, and elongation factor 1 alpha $(E F 1 \alpha)$, to assess their expression stability in different tissues and at different developmental stages using RT-qPCR. The expression stability and suitability of these genes for the normalization of RT-qPCR data were validated using the geNorm and NormFinder software packages. The results of this study provide useful reference information for the selection of appropriate reference genes for gene expression studies in persimmon.

\section{MATERIALS AND METHODS}

\section{Plant materials}

To determine the expression profiles of candidate genes from different tissues, we collected the roots, stems, leaves, female floral buds, fruits, and seeds of 10-year-old monoecious $D$. kaki "Zenjimaru." To determine the expression profiles of candidate genes at different developmental stages, female and male floral buds from dioecious $D$. kaki "Jiangxi wild persimmon" were collected at five important developmental stages (bract primordia initiation on June 3, 2015; development/arrest in two lateral flower primordia on June 17, 2015; carpel primordia initiation on March 28, 2016; microsporocyte/megasporocyte initiation on April 17, 2016; and mature pollen/embryo sac formation on May 3, 2016). All persimmon materials were collected from Yuanyang County, Henan Province, China (34 $55^{\prime} 30^{\prime \prime}-34^{\circ} 56^{\prime} 45^{\prime \prime} \mathrm{N}$ and $\left.113^{\circ} 46^{\prime} 24^{\prime \prime}-113^{\circ} 47^{\prime} 59^{\prime \prime} \mathrm{E}\right)$. After collection in triplicate, all samples were immediately frozen in liquid nitrogen and stored at $-80{ }^{\circ} \mathrm{C}$ until further use.

\section{Extraction and cDNA synthesis}

The EZ-10 DNAaway RNA Miniprep Kit (Sangon Biotechnology Co., Shanghai, China) was used to extract RNA from all samples according to the manufacturer's instructions. RNA integrity was validated under ultraviolet light in a gel documentation system after electrophoresis on a $1.0 \%(\mathrm{w} / \mathrm{v})$ agarose gel containing ethidium bromide. Quality and concentration were examined using a NanoDrop ND-2000 spectrophotometer (Thermo Scientific, Waltham, MA, USA). RNA with an OD260/OD280 ratio of 1.9-2.1 and OD260/OD230 ratio of 2.0-2.6 were used for cDNA synthesis and further analysis. Individual RNA samples were subsequently stored in a freezer at $-80{ }^{\circ} \mathrm{C}$ for later analysis steps. For each sample, $1 \mu \mathrm{g}$ of total RNA was reverse transcribed using the PC18-TRUE 1st Strand cDNA Synthesis Kit (Aidlab, Beijing, China) according to the manufacturer's protocol. Newly synthesized cDNA was diluted to $50 \mathrm{ng} / \mu \mathrm{l}$ with $\mathrm{ddH}_{2} \mathrm{O}$ and stored at $-20{ }^{\circ} \mathrm{C}$ until RT-qPCR analysis.

\section{Primer design}

Six commonly used housekeeping genes, $A C T, 18 \operatorname{Sr} R N A$, $G A P D H, E F 1 \alpha, U B Q$, and $T U B$, were selected as candidate reference genes for internal controls for normalization of gene expression measurements. Based on the sequences obtained from D. kaki "Zenjimaru" transcriptome results in our laboratory, primers (Table 1) were designed from coding sequences using the Premier 5.0 software (Premier, Inc., Canada) at a melting temperature $(\mathrm{Tm})$ of $55-65{ }^{\circ} \mathrm{C}$, primer size of $18-24 \mathrm{bp}, \mathrm{GC}$ content of $40 \%-60 \%$, and product length of 100-250 bp.

\section{$R T-q P C R$}

We conducted a RT-qPCR assay to examine the mRNA expression stability of the six candidate genes. The reaction mixture was prepared with SYBR Green Dye (Kemix, Beijing, China) in a $20-\mu$ reaction volume containing $2 \mu \mathrm{l}$ cDNA template, $10 \mu \mathrm{l} 2 \mathrm{X}$ SYBR qPCR mix, $0.5 \mu \mathrm{l}$ of each


formed using a Bio-Rad CFX96 RT-PCR platform (Bio-Rad Laboratories, Hercules, CA, USA) under the following cycle conditions: an initial denaturation step of $95{ }^{\circ} \mathrm{C}$ for $2 \mathrm{~min}$, followed by 40 cycles of $95^{\circ} \mathrm{C}$ for $5 \mathrm{~s}$ and $60{ }^{\circ} \mathrm{C}$ for $30 \mathrm{~s}$, and a $55-98^{\circ} \mathrm{C}$ melt curve analysis. All reactions were conducted in biological and technical triplicate. A nontemplate control was also included in each PCR run for each primer pair. The final quantification cycle $(\mathrm{Cq})$ values were calculated and used to evaluate the stability of reference genes under our experimental conditions.

\section{Statistical analyses}

Two widely used Microsoft Excel-based software packages, geNorm and NormFinder, were used to analyze gene 
Reference genes for quantitative gene expression analyses in persimmon

Table 1. Primer sequences of the six candidate reference genes used as internal controls

\begin{tabular}{lllr}
\hline Gene & Forward primer sequence $\left(5^{\prime}-3^{\prime}\right)$ & Reverse primer sequence $\left(5^{\prime}-3^{\prime}\right)$ & Amplification length $(\mathrm{bp})$ \\
\hline$A C T$ & ACCACAGCTGAACGGGAAAT & GCTCAGCCCCAATGGTGATA & 157 \\
GAPDH & AGCTCTTCCACCTCTCCAGT & TGCTAGCTGCACAACCAACT & 157 \\
$18 S r R N A$ & GTTTCTCAGGCTCCCTCTCC & TTTCTGCCCTATCAACTTTCG & 101 \\
$E F 1 \alpha$ & CAAGAGGCCTTCAGACAAGC & ACCATGCCAGGTTTGAGAAC & 117 \\
$U B Q$ & TCCCTGCCCTTCACTTAT & TTGTTACTGGTGCTCCTC & 245 \\
$T U B$ & GGGCTTTGAATGTGGATG & CTTGGTCTTGATAGTGGC & 278 \\
\hline
\end{tabular}

Note. ACT: actin; GAPDH: glyceraldehyde-3-phosphate dehydrogenase; TUB: alpha-tubulin; 18SrRNA: 18S ribosomal RNA; UBQ: ubiquitin; $E F 1 \alpha$ : elongation factor 1 alpha.

expression stability following the manufacturer's instructions. After completion of RT-qPCR analysis, Cq values were obtained and data were converted to relative quantities using the following formula: $\mathrm{Q}=\mathrm{E}^{\Delta \mathrm{Cq}}$, where $\Delta \mathrm{Cq}=$ $\mathrm{Cq}_{\text {minimum }}-\mathrm{Cq}_{\text {sample }}$. These data were further analyzed using geNorm and NormFinder.

\section{RESULTS}

Variability of $m R N A$ expression levels

The six candidate genes (18SrRNA, ACT, EF1 $\alpha, G A P D H$, $T U B$, and $U B Q)$ were screened for normalization of gene expression measurements. All candidate reference genes exhibited smooth melting curves with a single peak, showing that the primers were highly specific. Gene amplification curves for each sample had good repeatability and high sensitivity, showing that the RT-qPCR results were accurate and reliable (Fig. 1). To compare the mRNA expression levels of candidate reference genes across all tested samples, the absolute $\mathrm{Cq}$ value of each gene was determined using RT-qPCR results, such that higher gene expression led to a smaller $\mathrm{Cq}$ value and vice versa. $\mathrm{Cq}$ values varied widely from 12 to 30 cycles (Fig. 2), with median $\mathrm{Cq}$ values between 11.0 and 30.8. Among the candidate reference genes, 18SrRNA showed the highest transcriptional level with a median $\mathrm{Cq}$ value of 19.2 , but exhibited the highest RNA expression range (14.6; Table 2). The coefficient of



Fig. 2. Distribution of quantification cycle $(\mathrm{Cq})$ values for the six candidate reference genes from all samples. Boxes indicate the 10th and 90th percentiles; lines indicate median values; whiskers indicate ranges

variation was $<10 \%$ for $E F 1 \alpha, G A P D H$, and $T U B$ (Table 2). The six reference genes showed irregular variation in their expression patterns, which may be attributed to variation in cell types and developmental stages among persimmon varieties.

\section{Expression profiles of candidate genes in different tissues}

As shown in Fig. 3A, the Cq values of the six genes ranged between 16.9 and 30.7 among different tissues. Of the six genes, $18 \operatorname{SrRNA}$ had the lowest $\mathrm{Cq}$ values in all tissues types, ranging between 16.9 and 22.8. On the contrary, $U B Q$ exhibited the lowest gene expression in all tissue types
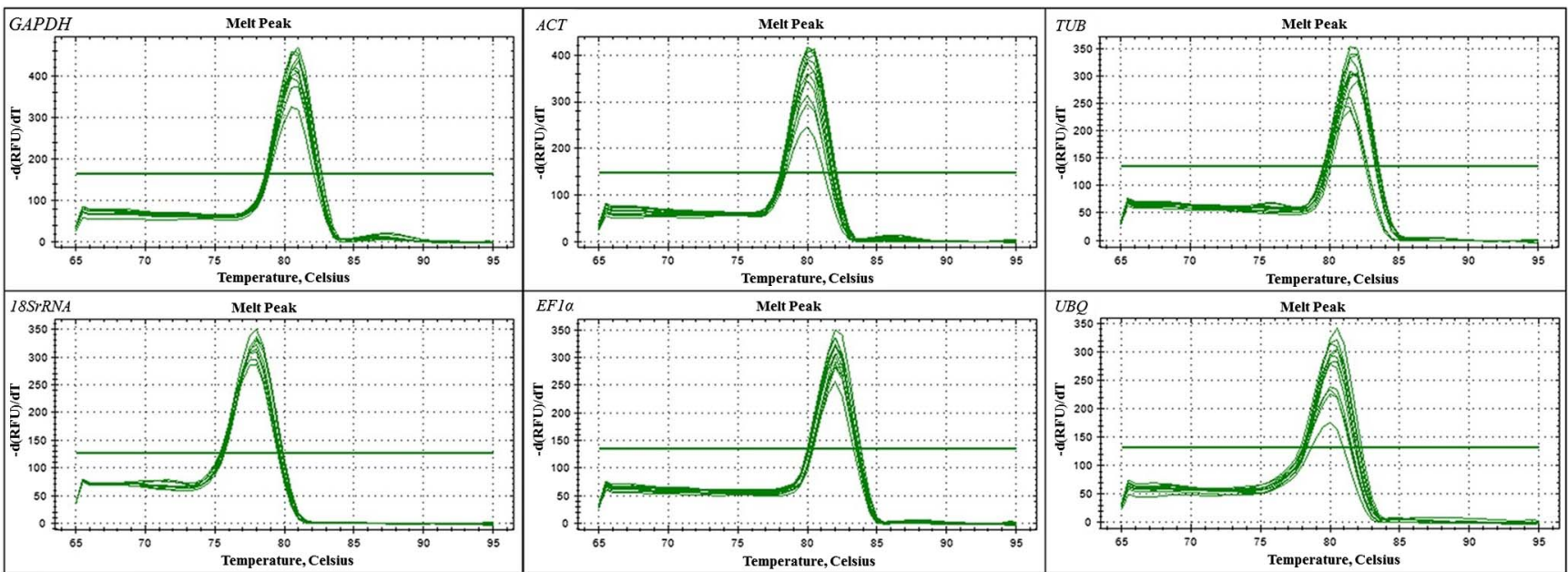

Fig. 1. Melting curves of the six genes generated for all amplicons 
Table 2. Summarized quantification cycle values of candidate genes from all tested samples

\begin{tabular}{lcccrr}
\hline Gene & $N$ & Average & $S D$ & Range & CV (\%) \\
\hline I8SrRNA & 60 & 18.711 & 3.883 & 14.620 & 20.752 \\
ACT & 60 & 24.457 & 3.780 & 7.830 & 15.454 \\
EF1 $\alpha$ & 60 & 22.573 & 2.059 & 9.840 & 9.120 \\
GAPDH & 60 & 22.843 & 2.137 & 8.400 & 9.354 \\
TUB & 60 & 25.907 & 1.706 & 6.800 & 6.585 \\
UBQ & 60 & 26.269 & 6.452 & 6.270 & 24.563 \\
\hline
\end{tabular}

Note. $N$ denotes total number of samples. Range denotes difference between maximum and minimum $\mathrm{Cq}$ values. $S D$ : standard deviation; CV: coefficient of variation; $A C T$ : actin; $G A P D H$ : glyceraldehyde-3-phosphate dehydrogenase; TUB: alpha-tubulin; 18SrRNA: 18S ribosomal RNA; UBQ: ubiquitin; EF1 $\alpha$ : elongation factor 1 alpha.

excluding stem tissue, with $\mathrm{Cq}$ values ranging from 25.6 to 30.7. The second most highly expressed genes were $E F 1 \alpha$ and $G A P D H$, which had similar expression profiles.

\section{Developmental expression profiles of candidate reference genes}

To better understand gene expression throughout reproductive development, female and male floral buds were collected from dioecious $D$. kaki "Jiangxi wild persimmon" at five important developmental stages. Transcripts of the six genes were detected in these samples by RT-qPCR. As shown in Fig. $3 \mathrm{~B}$ and $\mathrm{C}, \mathrm{Cq}$ values of the genes ranged from 13.4 to 30.3; the other results were quite similar to those obtained for $\mathrm{Cq}$ values among tissues. For example, $18 \operatorname{SrRNA}$ was the most highly expressed gene, with the highest expression range in both female and male floral buds. $U B Q$ was observed to have the lowest expression levels in both female and male floral buds. EFI $\alpha$ and GAPDH showed relatively similar expression profiles.

\section{Analysis of gene expression stability}

To investigate the stability values of the six candidate reference genes, both geNorm and NormFinder methods were applied in this study.

The geNorm software was applied to determine the gene expression stability of the selected reference genes (Andersen et al., 2004). $M$ values were calculated by the software, with higher $M$ values indicating lower expression stability. The $M$ values of the six candidate genes among different sample groups are presented in Fig. $4 \mathrm{~A}-\mathrm{C}$. $18 \operatorname{Sr} R N A$ consistently exhibited the highest $M$ values among all sample groups. The geNorm identified GAPDH as the most stable gene among tissue samples and male floral buds collected at different developmental stages (Fig. 4A and B). Among female floral buds collected at different developmental stages, $E F 1 \alpha$ was the most stable gene (Fig. 4C).

NormFinder (Vandesompele et al., 2002) is a Microsoft Excel-based Visual Basic application that ranks genes according to their stability under different experimental conditions. The results were consistent with those obtained by geNorm (Fig. 4D-F), such that gene expressional stability was negatively correlated with expression stability $(M)$.

\section{DISCUSSION}

Gene expression analysis is an efficient method to clarify the related traits of organisms. The application of RT-qPCR in this research field has improved the sensitivity and quantification of gene expression profiles (Andersen et al., 2004; Vandesompele et al., 2002). The selection of an appropriate internal control gene for data normalization is a crucial prerequisite for obtaining highly accurate results in RT-qPCR analysis. The ideal control gene must retain relatively stable and uniform expression among different organs, developmental stages, and experimental treatments (McCurley \& Callard, 2008). Using inaccurate reference genes can deflect gene expression profiles, leading to misguiding results (Chi et al., 2012). Therefore, a systematic verification of the most suitable reference gene as the internal control for accurate quantification of gene expression under specific experimental conditions is imperative. To identify the best reference genes for different organs and developmental stages in various persimmon varieties, geNorm and NormFinder were used in this study to determine the expression stability of six candidate genes.

Persimmon has complex flowering characteristics, with bisexual (hermaphrodite) and unisexual (male and female) flowers (Zhang, 2006). Most persimmon varieties are gynoecious, whereas a few are monoecious, and androecious specimens are extremely rare (Yonemori et al., 1993; Zhang et al., 2009; Xu et al., 2013). DNA methylation level of a promoter of the sex determination gene MeGI
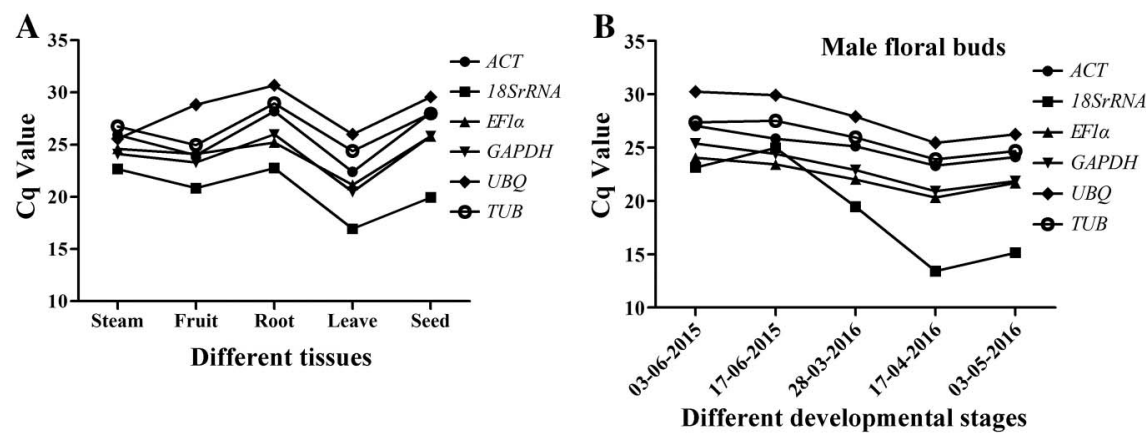

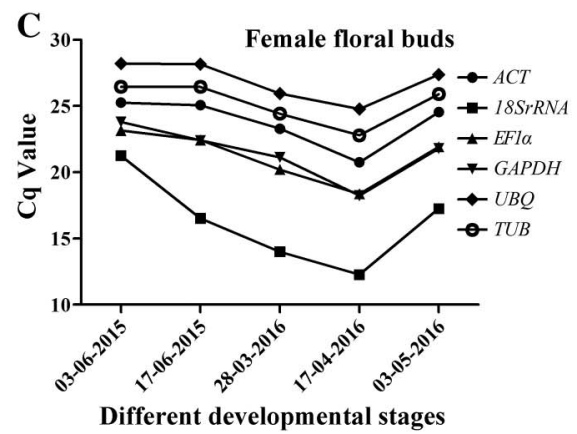

Fig. 3. Average Cq values of the six candidate reference genes among different tissues (A) and in male floral buds (B) and female floral buds (C) at different developmental stages 
A



$\mathbf{D}_{1.1}$

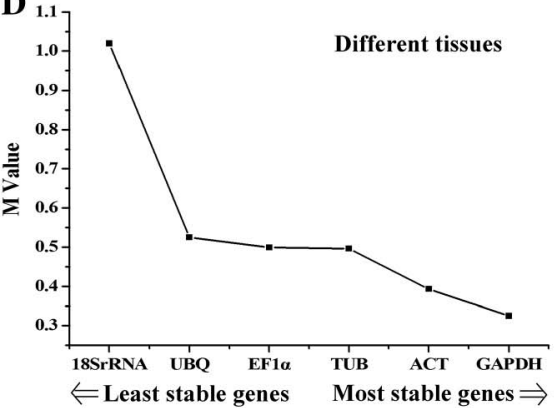

B



$\mathbf{E}$

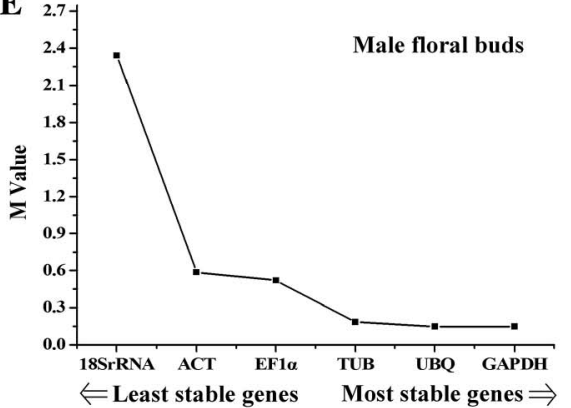

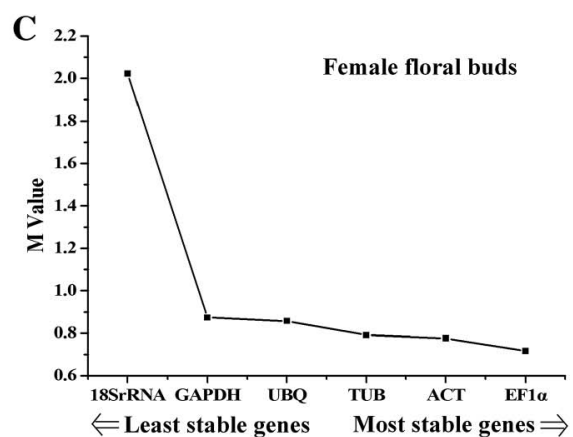

F

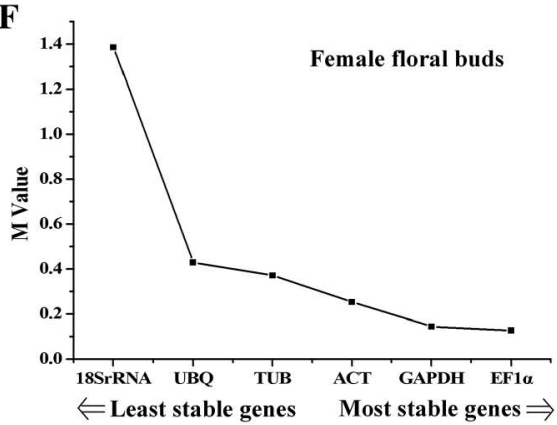

Fig. 4. Average expression stability values $(M)$ of the six reference genes calculated using geNorm software and NormFinder for different tissues (A and D) and for male floral buds (B and E) and female floral buds (C and F) at different developmental stages

determines the sexuality in persimmons, and low levels lead to the development of female flowers (Akagi et al., 2016). In addition, the male-linked gene locus $(O G I)$ was shown to be tightly linked to the maleness expression in persimmons (Zhang et al., 2016). As a result, we can distinguish flower sexuality using the OGI/MeGI system served as an early sex diagnosis technology for future investigations in persimmon (Kajita et al., 2015; Zhang et al., 2016). Moreover, our previous study showed that high levels genes homologous to IAA32, beta-amyrin 28-oxidase-like, and GA20OX2 may stimulate the development of male floral buds, while the genes homologous to $M e G I$ and $A C O$ showed female promoting effects (Li et al., 2019). Therefore, it is necessary to detect optimal internal control genes for male and female buds separately for data analysis accurately. Based on our statistical analyses, there were large differences in reference gene expression stability between male and female floral buds collected at different developmental stages. In male floral buds, GAPDH exhibited more stable expression, and among female floral buds, EFl $\alpha$ was more stable. To minimize error, it is highly recommended that different reference genes be considered for persimmon gene expression analysis by RT-qPCR if male and female floral buds are studied.

Several approaches have been used to assess the expression stability of reference genes; among these, geNorm and NormFinder are widely used analysis tools (Jain et al., 2006; Schmittgen \& Zakrajsek, 2000). When we compared the results obtained with each software application, we observed variation and consistency among the resulting ranks of stable reference genes. These findings may be due to the different analytical procedures employed by the two programs (Fu et al., 2013; Migocka \& Papierniak, 2011).

Among the six candidate reference genes, both geNorm and NormFinder found that $G A P D H$ retained perfectly stable expression among tissues sample groups and groups of male floral buds collected at different developmental stages, whereas $E F 1 \alpha$ was the most stable gene among groups of female floral buds collected at different developmental stages. According to NormFinder, $18 \operatorname{SrRNA}$ had the lowest stability values across all samples, which was consistent with the results produced by geNorm. On the basis of the analysis results from both algorithms, we determined that $G A P D H$ was the best reference gene for persimmon, with perfectly stable expression for data normalization among whole samples, followed by EF1 $\alpha$. These results are in agreement with those of previous studies (Han et al., 2012; Sun et al., 2010). Another prominent finding of this study is that $18 \operatorname{SrRNA}$ consistently showed the least stable gene expression. This result differed from those reported in other species (Blanquicett et al., 2002; Bond et al., 2002; Radonić et al., 2004; Schmittgen \& Zakrajsek, 2000). The findings of this study provide greater insight into the optimal control genes for the analysis of different growth stages and various organs among persimmon varieties.

\section{CONCLUSION FOR FUTURE BIOLOGY}

We systematically assessed reference genes for the normalization of RT-qPCR data in persimmon under different conditions. A stability analysis of gene expression by geNorm and NormFinder revealed that $G A P D H$ was the most stably expressed gene, and we recommend it as the appropriate reference gene for expression analysis in persimmon. To our knowledge, this is the first study to identify and validate suitable reference genes for persimmon using RT-qPCR analysis. This study will contribute in improving the accuracy of persimmon gene expression analysis results 
in various experimental settings, thus improving our understanding of the molecular mechanisms of sex differentiation and characterization of gene functions.

Funding Statement: This paper was supported by grants from the Fundamental Research Funds for the Central NonProfit Research Institution of CAF (CAFYBB2018QA009 and CAFYBB2017ZA004-3) and the National Natural Science Foundation of China (31500559).

Data Accessibility: The transcriptome sequencing raw data of D. kaki "Zenjimaru" were deposited in the National Center for Biotechnology Information Sequence Read Archive (NCBI SRA) under the accession number SRP151715.

Competing Interests: The authors declare no conflict of interest.

Authors' Contributions: PS and JF conceived and designed the experiments. GD and LW carried out the experiments, wrote the manuscript, and contributed equally to this study. HL, YS, WH, SD, YM, and FL helped to draft and review the manuscript. All authors read and approved the final version of the manuscript.

\section{REFERENCES}

Akagi, T., Henry, I. M., Kawai, T., Comai, L., Tao, R. (2016) Epigenetic regulation of the sex determination gene MeGI in polyploid persimmon. Plant Cell. 28, 2905-2915.

Andersen, C. L., Jensen, J. L., Ørntoft, T. F. (2004) Normalization of real-time quantitative reverse transcription-PCR data: a model-based variance estimation approach to identify genes suited for normalization, applied to bladder and colon cancer data sets. Cancer Res. 64, 5245-5250.

Blanquicett, C., Johnson, M. R., Heslin, M., Diasio, R. B. (2002) Housekeeping gene variability in normal and carcinomatous colorectal and liver tissues: applications in pharmacogenomic gene expression studies. Anal. Biochem. 303, 209-214.

Bond, B. C., Virley, D. J., Cairns, N. J., Hunter, A. J., Moore, G. B., Moss, S. J., Mudge, A. W., Walsh, F. S., Jazin, E., Preece, P. (2002) The quantification of gene expression in an animal model of brain ischaemia using TaqMan real-time RT-PCR. Mol. Brain Res. 106, 101-116.

Chen, L., Zhong, H. Y., Kuang, J. F., Li, J. G., Lu, W. J., Chen, J. Y. (2011) Validation of reference genes for RT-qPCR studies of gene expression in banana fruit under different experimental conditions. Planta 234, 377.

Chi, X., Hu, R., Yang, Q., Zhang, X., Pan, L. (2012) Validation of reference genes for gene expression studies in peanut by quantitative real-time RT-PCR. Mol. Genet. Genomics 287, 167-176.

Demidenko, N. V., Logacheva, M. D., Penin, A. A. (2011) Selection and validation of reference genes for quantitative real-time PCR in buckwheat (Fagopyrum esculentum) based on transcriptome sequence data. PLoS One 6, e19434.

Fu, J., Wang, Y., Huang, H., Zhang, C., Dai, S. (2013) Reference gene selection for RT-qPCR analysis of Chrysanthemum lavandulifolium during its flowering stages. Mol. Breed. 31, 205-215.
Guo, D. L., Luo, Z. R. (2006) Genetic relationships of some PCNA persimmons (Diospyros kaki Thunb.) from China and Japan revealed by SRAP analysis. Genet. Resour. Crop Evol. 53, 1597.

Han, X., Lu, M., Chen, Y., Zhan, Z., Cui, Q., Wang, Y. (2012) Selection of reliable reference genes for gene expression studies using real-time PCR in tung tree during seed development. PLoS One 7, e43084.

Hu, R., Fan, C., Li, H., Zhang, Q., Fu, Y. F. (2009) Evaluation of putative reference genes for gene expression normalization in soybean by quantitative real-time RT-PCR. BMC Mol. Biol. $10,93$.

Jain, M., Nijhawan, A., Tyagi, A. K., Khurana, J. P. (2006) Validation of housekeeping genes as internal control for studying gene expression in rice by quantitative real-time PCR. Biochem. Biophys. Res. Commun. 345, 646-651.

Jin, X., Fu, J., Dai, S., Sun, Y., Hong, Y. (2013) Reference gene selection for qPCR analysis in cineraria developing flowers. Sci. Hortic. 153, 64-70.

Kajita, K., Akagi, T., Yamane, H., Tao, R., Yonemori, K. (2015) The relationship between a maleness-associated region in Diospyrsos lotus L. and maleness of persimmon (D. kaki Thunb.) cultivars. Hortic. Res. 14, 121-126. (in Japanese with English summary)

Li, J., Chen, M., Qiu, F., Qin, B., Liu, W., Wu, N., Lan, X., Wang, Q., Liao, Z., Tang, K. (2014) Reference gene selection for gene expression studies using quantitative real-time PCR normalization in Atropa belladonna. Plant Mol. Biol. Rep. 32, 1002-1014.

Li, S. Z., Sun, P., Du, G. G., Wang, L. Y., Li, H. W., Fu, J. M., Suo, Y. J., Han, W. J., Diao, S. F., Mai, Y. N., Li, F. D. (2019) Transcriptome sequencing and comparative analysis between male and female floral buds of the persimmon (Diospyros kaki Thunb.). Sci. Hortic. 246, 987-997.

McCurley, A. T., Callard, G. V. (2008) Characterization of housekeeping genes in zebrafish: male-female differences and effects of tissue type, developmental stage and chemical treatment. BMC Mol. Biol. 9, 102.

Migocka, M., Papierniak, A. (2011) Identification of suitable reference genes for studying gene expression in cucumber plants subjected to abiotic stress and growth regulators. Mol. Breed. 28, 343-357.

Nicot, N., Hausman, J. F., Hoffmann, L., Evers, D. (2005) Housekeeping gene selection for real-time RT-PCR normalization in potato during biotic and abiotic stress. J. Exp. Bot. 56, 2907-2914.

Radonić, A., Thulke, S., Mackay, I. M., Landt, O., Siegert, W., Nitsche, A. (2004) Guideline to reference gene selection for quantitative real-time PCR. Biochem. Biophys. Res. Commun. 313, 856-862.

Schmittgen, T. D., Zakrajsek, B. A. (2000) Effect of experimental treatment on housekeeping gene expression: validation by realtime, quantitative RT-PCR. J. Biochem. Biophys. Methods 46, 69-81.

Sun, M. L., Wang, Y. S., Yang, D. Q., Wei, C. L., Gao, L. P., Xia, T., Shan, Y., Luo, Y. (2010) Reference genes for real-time fluorescence quantitative PCR in Camellia sinensis. Chin. Bull. Bot. 579-587.

Taira, S. (1996) Astringency in persimmon. In: Linskens, H. F., Jackson, J. F. (eds.) Fruit Analysis. Modern Methods of Plant Analysis, Springer, Berlin, Heidelberg, Vol. 18, pp. 97-110.

Teste, M. A., Duquenne, M., François, J. M., Parrou, J. L. (2009) Validation of reference genes for quantitative expression 
analysis by real-time RT-PCR in Saccharomyces cerevisiae. BMC Mol. Biol. 10, 99.

Tong, Z., Gao, Z., Wang, F., Zhou, J., Zhang, Z. (2009) Selection of reliable reference genes for gene expression studies in peach using real-time PCR. BMC Mol. Biol. 10, 71.

Vandesompele, J., Preter, D. K., Pattyn, F., Poppe, B., Roy, N. V., Paepe, D. A., Speleman, F. (2002) Accurate normalization of real-time quantitative RT-PCR data by geometric averaging of multiple internal control genes. Genome Biol. 3, research0034.1.

Xu, L. Q., Zhang, Q. L., Luo, Z. R. (2013) Pollen-related characteristics of Diospyros Linn. (Ebenaceae) androecious germplasms newly found in China. Acta Hortic. 996, 199-206.

Yin, X. R., Shi, Y. N., Min, T., Luo, Z. R., Yao, Y. C., Xu, Q., Ferguson, I., Chen, K. S. (2012) Expression of ethylene response genes during persimmon fruit astringency removal. Planta 235, 895-906.

Yonemori, K., Sugiura, A., Tanaka, K., Kameda, K. (1993) Floral ontogeny and sex determination in monoecious-type persimmons. J. Am. Soc. Hortic. Sci. 118, 293-297.

Zhang, Q. L. (2006) Genetic Relationships Between Pollination Constant Non-Astringent Persimmons and Some Staminate
Germplasm Native to China Based on RAPD and ISSR Analysis (PhD dissertation). Huazhong Agricultural University, Wuhan.

Zhang, P. X., Yang, S. C., Liu, Y. F., Zhang, Q. L., Xu, L. Q., Luo, Z. R. (2016) Validation of a male-linked gene locus (OGI) for sex identification in persimmon (Diospyros kaki Thunb.) and its application in F1 progeny. Plant Breed. 135, 721-727.

Zhang, Q. L., Guo, D. L., Luo, Z. R. (2009) Identification and taxonomic status of Chinese Diospyros spp. (Ebenaceae) androecious germplasms. Acta Hortic. 833, 91-96.

Zhao, D., Zhou, C., Sheng, Y., Liang, G., Tao, J. (2011a) Molecular cloning and expression of phytoene synthase, lycopene betacyclase, and beta-carotene hydroxylase genes in persimmon (Diospyros kaki L.) fruits. Plant Mol. Biol. Rep. 29, 345-351.

Zhao, D., Zhou, C., Tao, J. (2011b) Carotenoid accumulation and carotenogenic genes expression during two types of persimmon fruit (Diospyros kaki L.) development. Plant Mol. Biol. Rep. 29, 646-654.

Zhou, L., Zhang, L. Y., Zhang, C. X. (2012) Screening of reference genes for real-time fluorescence quantitative PCR in apple (Malus domestica). J. Fruit Sci. 29, 965-970. 